\title{
MODELLING OF ARTERIAL BIFURCATION BY MEANS OF ELECTROMECHANICAL MODEL WITH DISTRIBUTED PARAMETERS
}

The present paper deals with the computer-aided modelling of blood vessels bifurcations using the electro-mechanical analogy with the electric transmission lines. The present model allows assessing the Murray's cubic law and its comparison with the real state of large and small arteries branching in the human body.

\section{Introduction}

Knowledge of detailed mechanisms of blood circulation in a human body is important in many diseases diagnoses. Very effective way to obtain useful information is provided by computer-aided modelling of cardiovascular system. Many models are based on the electromechanical analogy. The recent models using analogy of the blood vessel with an electrical transmission line with distributed parameters are connected with modern powerful computers, which are able to perform extremely demanding mathematical calculation. On the basis of theoretical analysis [1] we have developed model of the human arterial system in the MATLAB environment [2]. This model allows to simulate different phenomena taking place in the arterial system and to search for proper medical diagnostic methods, e.g. [3]. We have found direct connection between the blood pressure wave velocity and the properties of the blood vessel [4]. In the present paper modeling of arterial bifurcation and its influence on the blood flow is demonstrated.

\section{Theory}

The theoretical model resulting from the electro-mechanical analogy describes the flow of blood in a vessel taken as an elastic tube. The blood flow is taken as the laminar flow of a viscous liquid. The tube with liquid is modelled by an electrical transmission line [1], [2], the elementary segment of which can be substituted by the longitudinal impedance $Z_{L}$ and transverse admittance $Y_{T}$ given by

$$
Z_{L}=-\frac{j \omega \rho J_{0}\left(a r_{0}\right)}{\pi r_{0}^{2} J_{2}\left(a r_{0}\right)} \text { and } Y_{T}=j \omega \frac{2 \pi r_{0}}{k_{w}\left(E_{w}+j \omega \eta_{w}\right)}
$$

where $a=\sqrt{-\frac{j \omega \rho}{\eta}}$ is the blood parameter, $\rho$ the blood density, $\eta$ the blood viscosity, $\omega$ the angular frequency of the transmitted wave, $r_{0}$ the tube internal radius, $E_{w}$ and $\eta_{w}$ the tube wall elastic modulus and tissue viscosity and $k_{w}$ the dimension factor. $J_{0}$ and $J_{2}$ are the Bessel functions of the 0 -th and 2-nd orders. According to the transmission line theory, the quantities (1) allow to express the complex propagation constant $\mathrm{k}$ and the complex wave impedance $Z_{0}$

$$
k=\sqrt{Z_{L} Y_{T}} \text { and } Z_{0}=\sqrt{\frac{Z_{L}}{Y_{T}}}
$$

The secondary parameters $\boldsymbol{k}$ and $\boldsymbol{Z}_{0}$ are important for the investigation of propagation of the blood pressure and blood flow waves in the vascular system.

\section{Investigation on the arterial bifurcation}

Cardiovascular system is very complex and widely bifurcated system, the primary function of which is to supplement oxygen and nutrients to every cell of the human body. From the heart to the distal parts the arteries bifurcate and build more complex system. Each arterial embranchment, Fig. 1, influences the blood pressure and blood flow waveforms and causes their changes in various parts of the circulatory system. In the electromechanical analogy model of arterial bifurcation the branching part represents the impedance mismatch which causes the blood pressure and blood flow wave reflections. Thus the final shape of the blood pressure and blood flow waveforms in each vessel are the result of the summation of the primary wave and reflected waves. In general, the waves up to order 20-th harmonic (reflected from the bifurcations and also

\footnotetext{
* Barbora Czippelova, Daniela Gombarska

Faculty of Electrical Engineering, University of Zilina, Slovakia, E-mail: gombarska@fel.uniza.sk
} 
from the source of the signal) contribute to the final shape of the waveform [5].

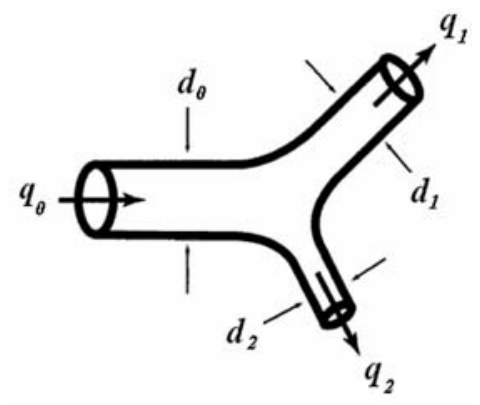

Fig. 1. Blood vessel branching

Basically two elementary conditions are valid for the arterial branching:

- The law of mass conservation - the blood flow in the mother vessel is equal to the sum of blood flows in daughter vessels

$$
q_{0}=q_{1}+q_{2}
$$

- In laminar blood flow case (turbulent flow on the bifurcation does not arise), the blood pressure is equal in all vessels in the place of branching

$$
p_{0}=p_{1}=p_{2}
$$

In order to investigate the influence of the branching vessels geometrical properties on the blood pressure and blood flow waveforms a simple arterial bifurcation is modelled by means of electromechanical model. The main interest is in the effect of the branching on the blood pressure and blood flow waveforms. Therefore al the reflection phenomena are located to the arterial branching which is achieved through used terminal segments; both daughter vessels are terminated with their characteristic impedance (for the 1st harmonic component).

The conditions (3) and (4) listed above imply that the combination of the daughter vessels is parallel and the impedance $\boldsymbol{Z}_{0 T}$, which represents the output impedance of the mother vessel, is given as parallel combination of the characteristic impedances of branching daughter vessels $\boldsymbol{Z}_{01}$ and $\boldsymbol{Z}_{02}$ as

$$
\frac{1}{Z_{0 T}}=\frac{1}{Z_{01}}+\frac{1}{Z_{02}} .
$$

The reflection coefficients $\boldsymbol{R}_{U}$ for blood pressure (equivalent to voltage) and $\boldsymbol{R}_{I}$ for the blood flow (equivalent to current) of basic vascular branching can be defined as

$$
\begin{aligned}
& R_{U}=\frac{Z_{0 T}-Z_{0 m}}{Z_{0 T}+Z_{0 m}} \\
& \boldsymbol{R}_{I}=\frac{Z_{0 T}-Z_{0 m}}{Z_{0 T}+Z_{0 m}}=-\dot{\boldsymbol{R}}_{U},
\end{aligned}
$$

where $\boldsymbol{Z}_{0 m}$ is the mother vessels' characteristic impedance.

Transmission of the energy through the arterial branching is the most effective if the absolute value of the reflection coefficient is minimal. Simple condition, which defines the vessel radii of the arterial branching for the most effective transmission of energy through the arterial bifurcation is given by the Murray's cubic law, e.g. [6] and [7]

$$
r_{0}^{3}=r_{1}^{3}+r_{2}^{3}
$$

The influence of the arterial branching on the haemodynamical quantities (blood pressure, blood flow) is investigated using the model in Fig. 2. The mother vessel is divided into 20 segments and both daughter vessels into 10 segments. Red arrows point to "measurement" sites.

The influence of the daughter vessels' radii on the blood pressure and blood flow waveforms is studied. All used geometrical and mechanical parameters of the vessels and of the blood are summarized in Tab. 1.

Table 1

\begin{tabular}{|l|c|}
\hline Diameter of mother vessel & $0.5 \mathrm{~cm}$ \\
\hline Length of the mother vessel & $30 \mathrm{~cm}$ \\
\hline Length of the daughter vessels & $15 \mathrm{~cm}$ \\
\hline Arterial wall thickness of the mother vessel & $0.67 \mathrm{~mm}$ \\
\hline Arterial wall thickness of the daughter vessels & $0.44 \mathrm{~mm}$ \\
\hline Terminal resistance & $1.44 \times 10^{10} \mathrm{~Pa} \cdot \mathrm{s} / \mathrm{m}^{3}$ \\
\hline Modulus of elasticity & $0.4 \mathrm{MPa}$ \\
\hline Blood density & $1.06 \times 10^{3} \mathrm{~kg} \cdot \mathrm{m}^{-3}$ \\
\hline Blood viscosity & $3.25 \times 10^{-3} \mathrm{~Pa} \cdot \mathrm{s}$ \\
\hline
\end{tabular}

Optimum radii of the daughter vessels appointed on the base of cubic law is $r^{*}=0.397 \mathrm{~cm}$. In order to investigate the influence of the daughter vessel radii on the blood flow dynamics daughter, the radius values were chosen as $r=0.357 \mathrm{~cm} ; 0.318 \mathrm{~cm} ; 0.278 \mathrm{~cm}$; $0.238 \mathrm{~cm}$ and $0.437 \mathrm{~cm} ; 0.476 \mathrm{~cm}$ (it means $90 \%, 80 \% 70 \%, 60 \%$ and $110 \%, 120 \%$ of $r^{*}$, respectively). Absolute values and the phases of the reflection coefficient for all values of the radius and for the 1st harmonic component are shown in Fig. 3.

The reflection coefficient achieves the minimum absolute value $\left|\boldsymbol{R}_{U}\right|=0.041$ in the case of the daughter vessels having the optimum radius $r^{*}$. In this case the phase achieves the value $\psi_{U}=$ $=-153^{\circ}$, which is in the transient area, where the phase changes from $0^{\circ}$ for radii less than $r^{*}$ down to the $-180^{\circ}$ for the radii greater than $r^{*}$. The absolute values of the reflection coefficient $\left|\boldsymbol{R}_{U}\right|$ for all other daughter vessel radii are greater than those for the optimum radius. For smaller diameters the reflected wave is in phase with the progressive pressure wave and the resultant blood pressure in the mother vessel increases. For greater radii the blood pressure wave reflects with opposite phase and the reflected 

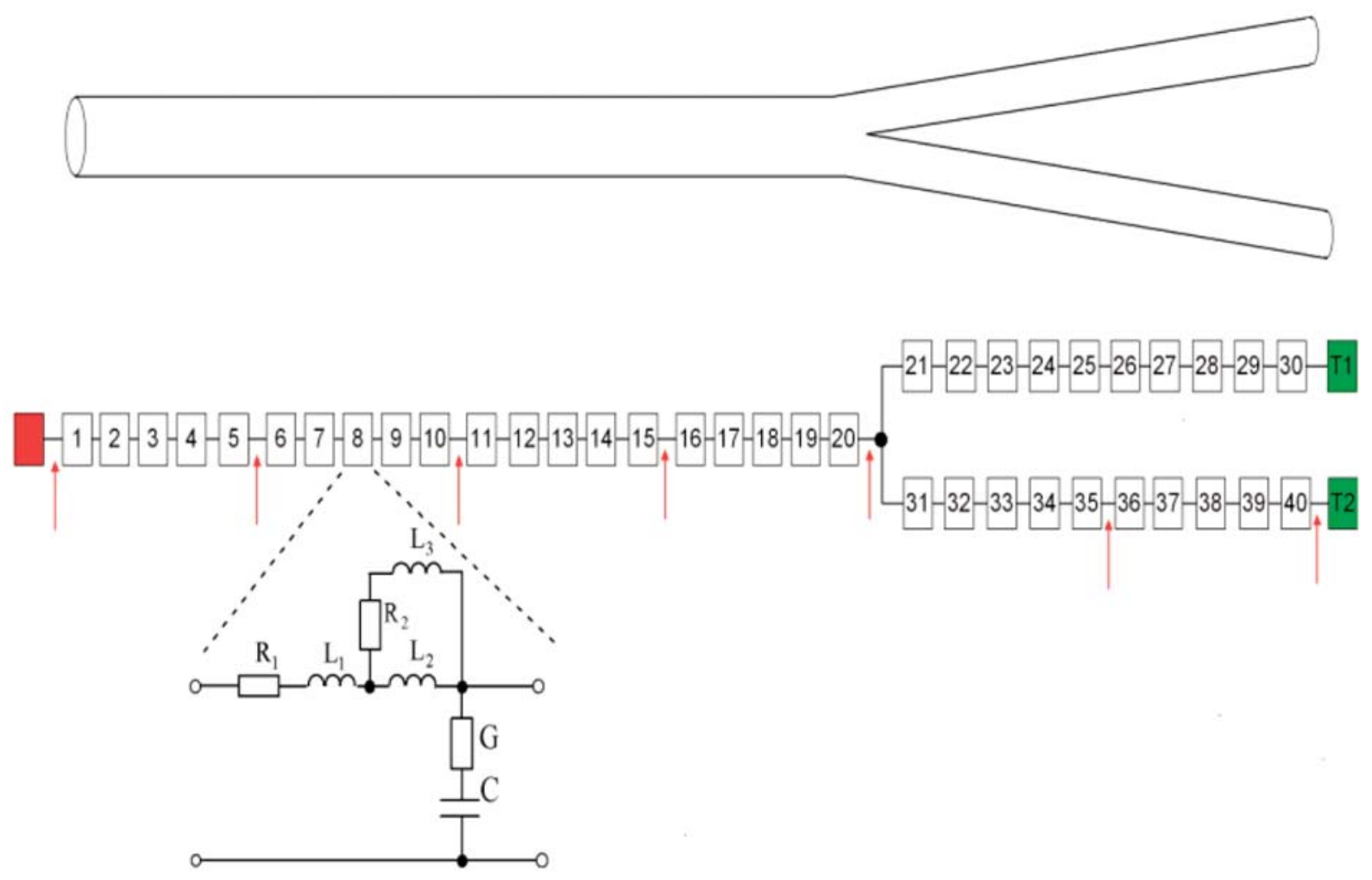

Fig. 2. Simulation of the vessel branching

Reflection coeficient- absolut value

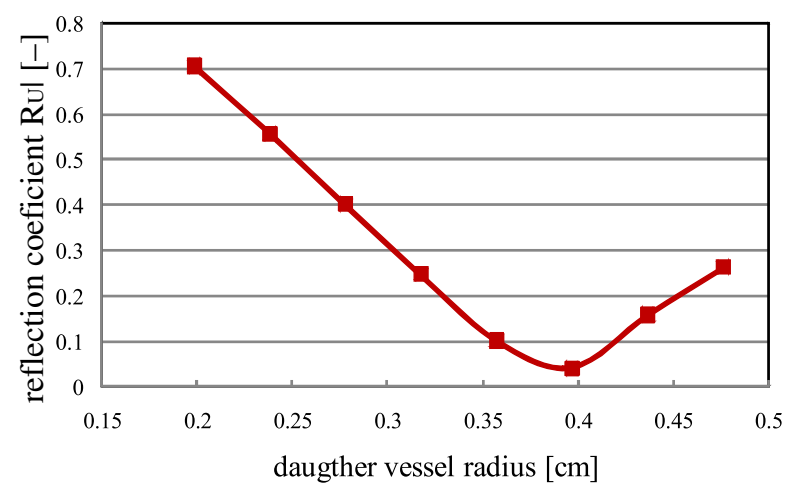

Reflection coeficient-phase

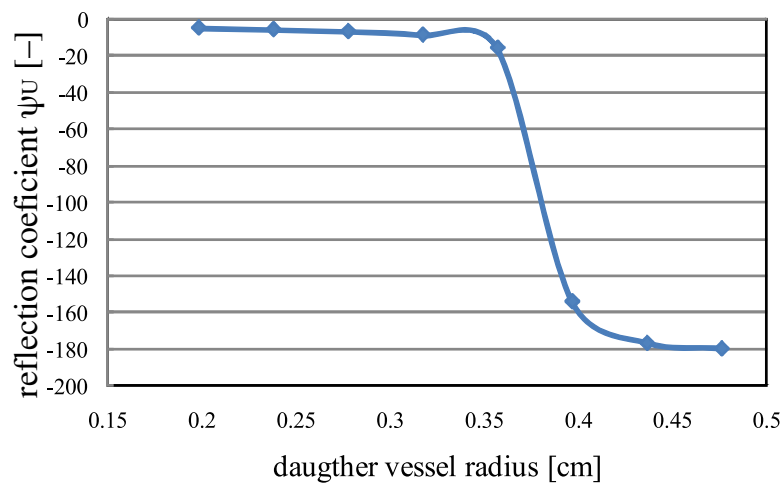

Fig. 3. Reflection coefficient of the blood pressure wave

wave suppresses the resultant blood pressure wave in the mother vessel. According to the (7): $\boldsymbol{R}_{I}=-\boldsymbol{R}_{U}$, therefore the resultant blood flow increases for greater daughter vessel radii and decreases for radii smaller than $r^{*}$. These facts proof the cube law.

All the facts listed above are depicted in detail in the figures 4 to 7 . The blood pressure magnitude for the biggest investigated daughter vessel radius $r=0.476 \mathrm{~cm}$ measured before branching reaches the value $113 \mathrm{mmHg}$ and the magnitude of blood flow is $4.17 \times 10^{-7} \mathrm{~m}^{3} / \mathrm{s}$. In case of the smallest investigated radius $r=$ $=0.238 \mathrm{~cm}$ the magnitude of blood pressure is $133.9 \mathrm{mmHg}$ and magnitude of blood flow $1.52 \times 10^{-7} \mathrm{~m}^{3} / \mathrm{s}$, Fig. 4. Besides changes in magnitude, the decrease in vessel radius implies also changes of blood pressure waveform, where the pulse profile can include up to three maxima for $r=0.238 \mathrm{~cm}$ due to the wave reflections. The results of blood flow and blood pressure time-dependence denote the significant influence of outgoing vessel diameter on the blood flow dynamics.

Figs. 5, 6 and 7 show the propagation of blood pressure and blood flow waves along mother vessel for three different radii. In Fig. 5 there are depicted pressure and flow waveforms for the radius calculated from the cubic law. It is evident that for this case the energy transmission is optimal. Fig. 6 shows the propagation of 

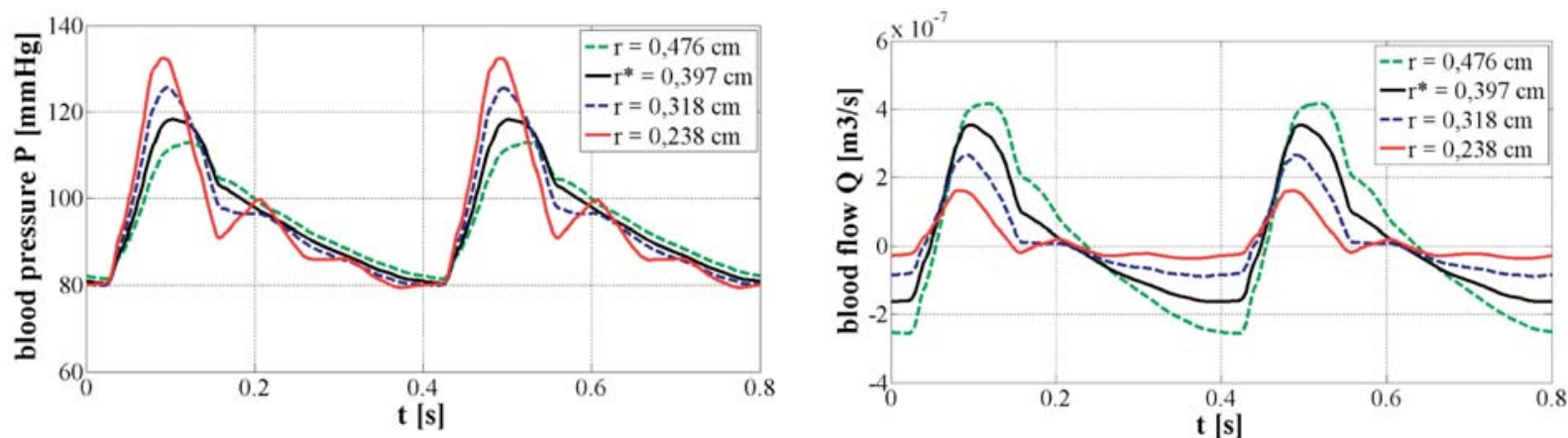

Fig. 4 Blood pressure and blood flow before branching for four different vessel radii
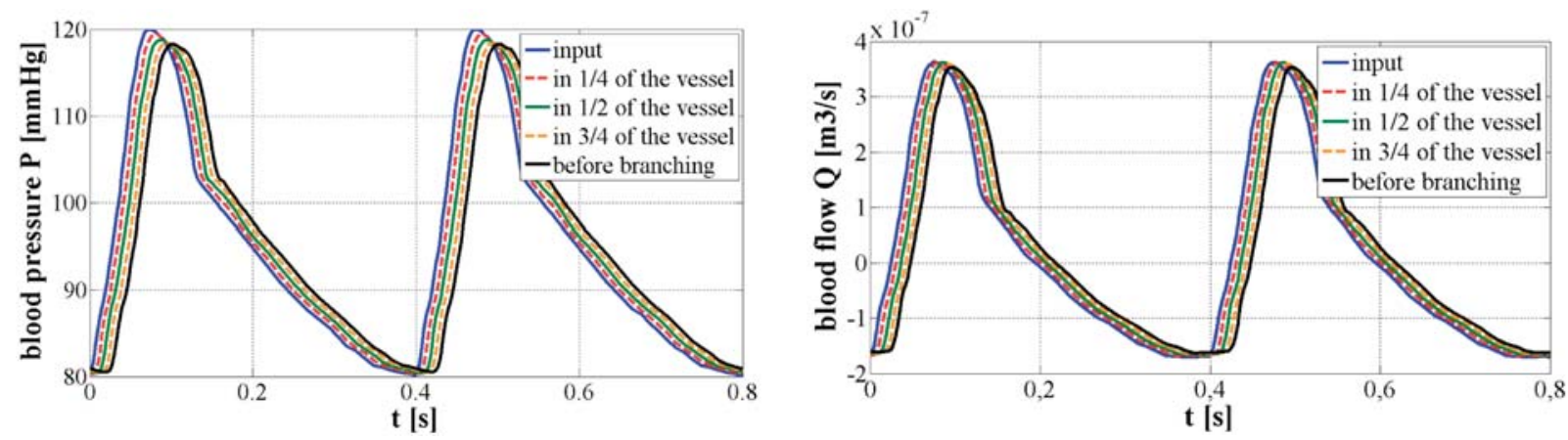

Fig. 5 Blood pressure and blood flow for $r^{*}=0.3 \mathrm{~cm}$
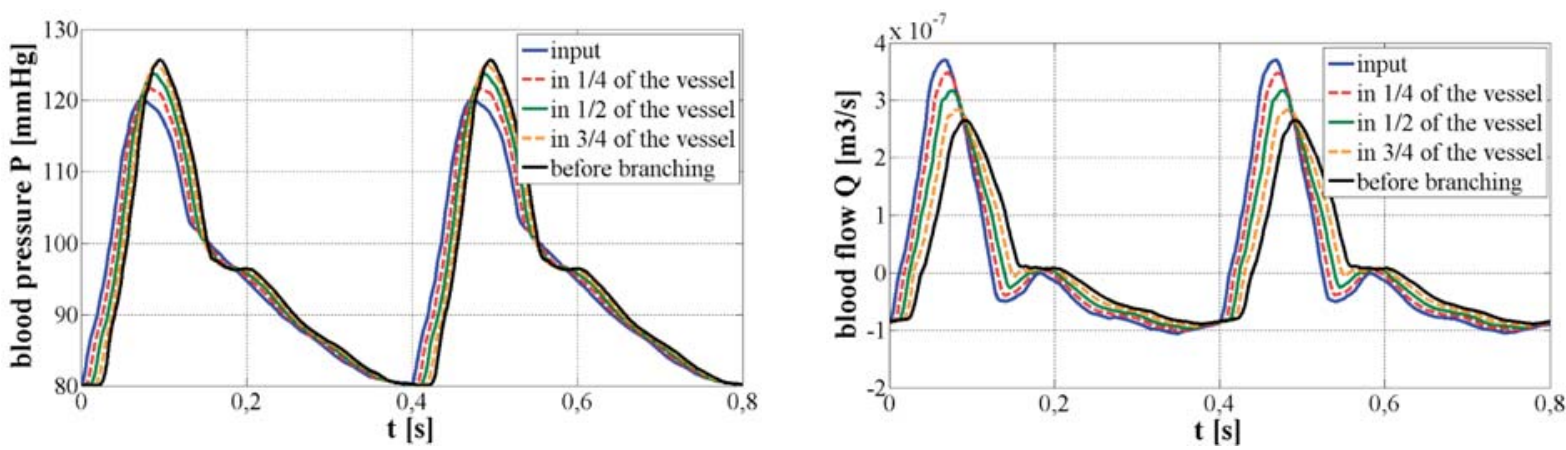

Fig. 6 Blood pressure and blood flow for $r=0.318 \mathrm{~cm}$
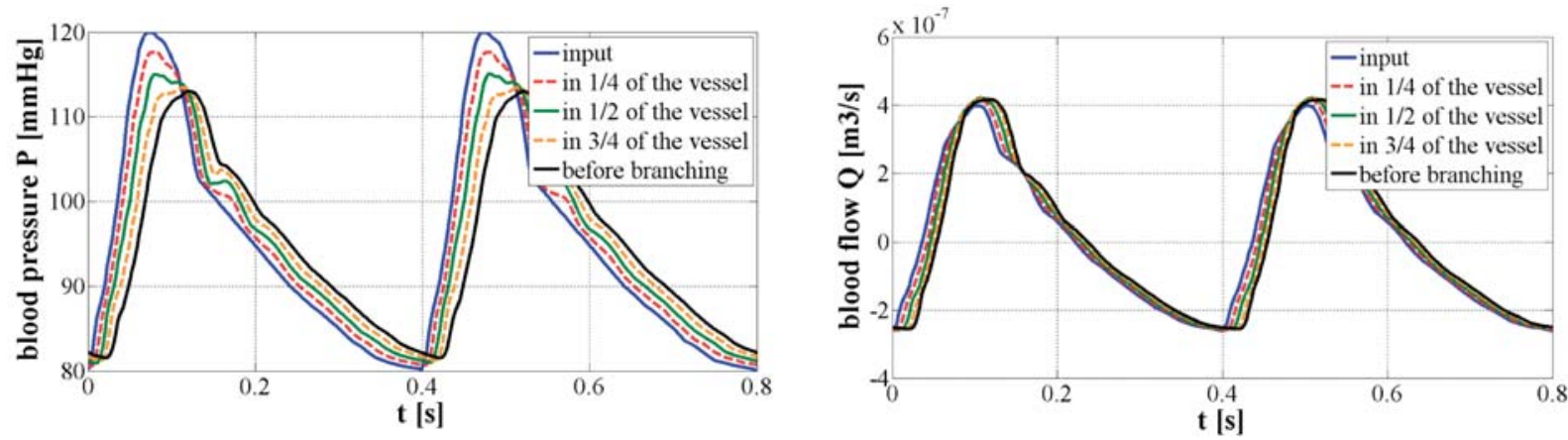

Fig. 7 Blood pressure and blood flow for $r=0.476 \mathrm{~cm}$ 
heamodynamic waves for daughter vessel diameter $r=0.318 \mathrm{~cm}$. Blood pressure increases along the artery and blood flow decreases due to reflections and also the second maximum appears in the waveforms. On the other side for the daughter vessel diameter bigger than $r^{*}(r=0.476 \mathrm{~cm})$ blood pressure wave decreases as it travels along the mother vessel whereas blood flow increases very slightly, Fig. 7.

In the cardiovascular system of the human body all three cases of bifurcation occur. Bigger arteries bifurcate in the way of $r_{0}^{3}<$ $<r_{1}^{3}+r_{2}^{3}$ (e.g. aorta $r=0.57 \mathrm{~cm}$ ) branches into two common iliac arteries $(r=0.52 \mathrm{~cm}))$, whereas peripheral arteries bifurcate in the way of $r_{0}^{3}><r_{1}^{3}+r_{2}^{3}$ (e.g. brachial artery $(r=0.24 \mathrm{~cm})$ branches into ulnar artery $(r=0.21 \mathrm{~cm})$ and radial artery $(r=$ $=0.16 \mathrm{~cm})$ ). Occasionally the branching with minimal reflection coefficient can be found (e. g. popliteal artery $(r=0.2 \mathrm{~cm})$ divides into anterior tibial artery $(r=0.13 \mathrm{~cm})$ and posterior tibial artery $(r=0.18 \mathrm{~cm}))$. As there is great amount of peripheral bifurcations in the cardiovascular system the influence of these branching points is dominant. It results in increasing the blood pressure towards the periphery and also in appearance of the second maximum in the blood pressure waveform in the more peripheral measured signals.

\section{Conclusion}

The present study describes the partial phenomenon of the vessels bifurcation, which is important for understanding reflections of blood pressure waves and their influence on the blood pressure pulse profile. The computer-aided model has approved the Murray's cubic law and it has described the deviations from this law in large and small arteries bifurcations. Modeling of physiological processes with help of modern powerful computers gives an effective tool for the present medicine. Simulation of different parts of a human body allows developing non-invasive diagnostic and prognostic methods supporting the treatment of various diseases.

\section{References}

[1] CAPOVA, K., BLAZEK, V., CAP, I., BUCKULIAKOVA, L.: Physiological fluid systems modelling and visualisation. International J. of Applied Electromagnetics and Mechanics 14 (2001/2002), IOS Press, pp. 377-380, ISSN 1383-5416

[2] CAPOVA, K., CAP, I., BLAZEK, V.: Brain Haemodynamics Modelling Using Electromagnetic Systems Characteristics. International J. of Applied Electromagnetics and Mechanics 19 (2004), No. 1-4, IOS Press, pp. 457-462. ISSN 1383-5416

[3] CAPOVA K., BLAZEK V., CAP I.: Investigation of Brain Arterial Circle Malformations Using Electrical Modelling and Simulation. Advances in Electrical and Electronic Engineering, Vol. 5 (2006), No. 1-2, pp. 212-217. ISSN 1336-1376

[4] CZIPPELOVA B., CAP I.: Pulse Wave Velocity Calculation by Means of Electromechanical Model of Blood Vessel, TRENDY V BMI 2009 - Bratislava, pp. 149-152, ISBN 978-80-227-3105-8

[5] CZIPPELOVA B., GOMBARSKA D.: Investigation of Blood Pressure and Blood Flow Wave Propagation on Arterial Bifurcation, Proc. of the IEEE 2009 - Králíky, pp. 44-47, ISBN 978-80-214-3938-2

[6] SHERMAN T. F.: On Connecting Large Vessels to Small - the Meaning of Murray’s law. J. Gen. Physiology, Vol. 78 (1981), pp. 431-453.

[7] REVELLIN R. et al.: Extension of Murray's law using a non-Newtonian model of blood flow. Theoretical Biology and Medical Modelling, 2009, 6:7 Open Access: http://www.tbiomed.com/ content/pdf/1742-4682-6-7.pdf . 